\title{
Catalytic Enantioselective Nucleophilic Desymmetrisation of Phosphonate
}

\section{Esters}

Authors: Michele Formica ${ }^{1}$, Tatiana Rogova ${ }^{1}$, Heyao Shi ${ }^{1}$, Naoto Sahara ${ }^{1,2}$, Alistair J. M. Farley ${ }^{1}$, Kirsten E. Christensen, ${ }^{1}$ Fernanda Duarte ${ }^{1 *} \&$ Darren J. Dixon ${ }^{1 *}$

Affiliation: ${ }^{1}$ Chemistry Research Laboratory, Department of Chemistry, University of Oxford, 12 Mansfield Road, Oxford, OX1 3TA, UK; ${ }^{2}$ Graduate School of Engineering, Nagoya University, B2-3(611), Furo-cho, Chikusa, Nagoya 464-8603, Japan

*e-mail:fernanda.duartegonzalez@chem.ox.ac.uk,darren.dixon@chem.ox.ac.uk

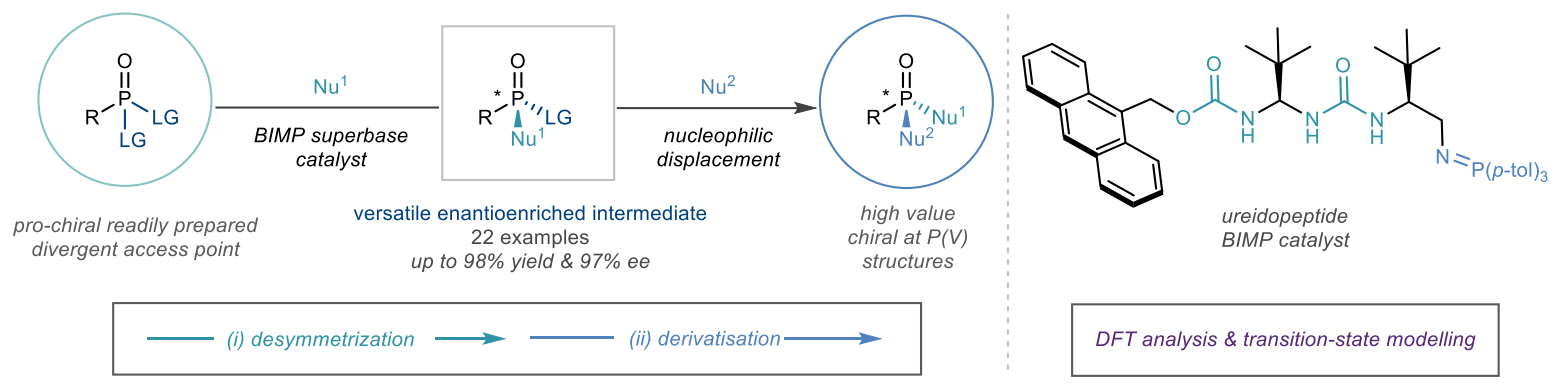

Compounds containing one or more stereogenic phosphorous atoms in the $P(V)$ oxidation state are important to chemistry, biology and medicine. ${ }^{1}$ These include marketed antiviral drugs such as Tenofovir alafenamide and Remdesivir, ${ }^{2}$ an effective treatment for Ebola which has also recently been approved for use against SARS-CoV-2 in the US. Existing approaches for the stereoselective synthesis of $\boldsymbol{P}$-stereogenic centers, while elegant, remain mostly diastereoselective, with catalytic enantioselective approaches remaining limited in application. Accordingly, conceptually novel, broad-scope, catalytic strategies for the efficient stereoselective synthesis of diverse stereogenic $\mathbf{P}(\mathrm{V})$ containing compounds remain essential. ${ }^{3}$ 
To this end, we describe a novel enantioselective two-stage strategy, exploiting the first catalytic and highly enantioselective ${ }^{4-6}$ desymmetrisation $^{7}$ of phosphonate esters. Pivoting on the stereocontrolled, sequential nucleophilic substitution of enantiotopic leaving groups from readily accessible pro-chiral $\mathbf{P}(\mathrm{V})$ precursors, a bifunctional iminophosphorane (BIMP) $)^{8-10}$ superbase catalyst was found to be essential in delivering reactive desymmetrised intermediates capable of downstream enantiospecific substitution. This uniquely modular, catalytic platform allows broad-scope, stereoselective access to a diverse library of chiral $\mathbf{P}(\mathrm{V})$ compounds including those with $O, N$ and $S$-linkages.

Over the past few years substantial progress has been made in improving the synthesis of $\mathrm{P}(\mathrm{V})$ chiral compounds. In 2017, Merck reported a diastereoselective dynamic kinetic asymmetric transformation (DYKAT) to synthesise MK-3682, a lead ProTide ${ }^{11}$ antiviral compound as well as other analogues. ${ }^{12}$ The process was reliant on a chiral C2 symmetric nucleophilic organocatalyst, which provided the desired prodrug with exquisite diastereocontrol from racemic $\mathrm{P}(\mathrm{V})$ starting material.

Recently, the Baran group reported a highly versatile chiral P(V) reagent: PSI ( $\psi)$, derived from limonene, for the diastereoselective synthesis of phosphorothioate oligonucleotides and of cyclic dinucleotides (CDNs). ${ }^{13}$ Building upon previous chiral auxiliary approaches, ${ }^{14-18}$ this work was also expanded towards the synthesis of $P$-chiral phosphines, as well as methylphosphonate oligonucleotides (MPOs). ${ }^{19}$ 
While these elegant processes towards $P$-chiral compounds are effective, they remain diastereoselective, where chirality at the phosphorous centre is installed either through a matched combination of catalyst and a carefully tailored starting material or by employing a stoichiometric chiral leaving group. Notwithstanding sophisticated, contemporary enantioselective arylation ${ }^{20,21}$ and allylation ${ }^{22}$ protocols of secondary phosphine oxides (limited to all carbon substituents on phosphorous), a few notable reports have also been disclosed generating a $P$-stereogenic centre indirectly through the functionalisation of enantiotopic $P$ bound groups. This approach however, inherently limits the scope and application of these protocols (Figure 1, A). ${ }^{3,23-33}$ Despite these impressive advances, to date, no catalytic enantioselective desymmetrisation protocols have been established with reactivity occurring directly at the phosphorous atom. ${ }^{34}$

We envisioned a novel, two-stage desymmetrisation-derivatisation strategy by which enantiotopic phenolic leaving groups on a pro-chiral phosphonate ester are enantiodiscriminated by a suitable nucleophile under the control of a chiral Brønsted base catalyst, ${ }^{35}$ generating a new $\mathrm{P}-\mathrm{O}$ bond. The resulting enantioenriched intermediate would retain suitable reactivity for sequential substitution of the remaining leaving group. With appropriate stereocontrol, this uniquely modular approach would overcome the key restrictions of previously developed protocols. Given the tunability and high basicity of our bifunctional iminophosphorane catalysts (BIMPs) $)^{8-10}$ we expected that they would provide sufficient pronucleophile / substrate activation and could be suitably adapted to obtain high levels of enantioselectivity in the products.

To explore the proposed reaction design, we began our investigations by identifying a suitable model system containing a phosphonyl dichloride mimic, utilising 2,4-dimethyl phenol as the 
nucleophile and a BIMP superbase catalyst. The leaving group had to possess key characteristics based on the dichotomy between leaving group ability / basicity and stability of the $\mathrm{P}(\mathrm{V})$ precursor to allow for reactivity and catalyst turnover. After a comprehensive investigation of potential leaving groups, para-nitrophenol was identified to possess good reactivity as well as crucially permitting catalyst turnover ${ }^{36}$ (see SI for details). Following this key breakthrough, a survey of nitrophenol isomers resulted in ortho-nitrophenol (LG1) being identified as optimal for enantioselectivity. The development of a new BIMP catalyst bearing a ureidopeptide H-bond donor motif (B1), inspired by the work of Palomo, ${ }^{37-39}$ and tuning of the reaction conditions led to a first lead result of $41 \%$ yield and $87 \%$ ee (Figure 1, B). To augment catalyst turnover, the $\mathrm{p} K_{\mathrm{a}}$ of the leaving group was carefully adjusted via a methyl group scan around the aromatic ring of the nitrophenol (LG2-LG4). While the introduction of a methyl group at the 4-position (LG3) gave the best yield (61\%), a methyl group at the 6position (LG4) gave optimal enantioselectivity (94\% ee) albeit with a drop in reactivity. By conducting the reaction at room temperature and fine-tuning the basicity of the catalyst (exchanging $\mathrm{PPh}_{3}$ to $\left.\mathrm{P}(p \text {-tol })_{3}\right)$ for the generation of the iminophosphorane), the yield of product 1 was increased to $84 \%$ and its enantiomeric excess was found to be $91 \%$. For comparison, it is worthy of note that Cinchona-derived bifunctional organocatalysts and phase transfer catalysis were ineffective in providing the desired product in high yield and enantioselectivity (see SI for details). 
A Enantioselective desymmetrisation at $P(V)$

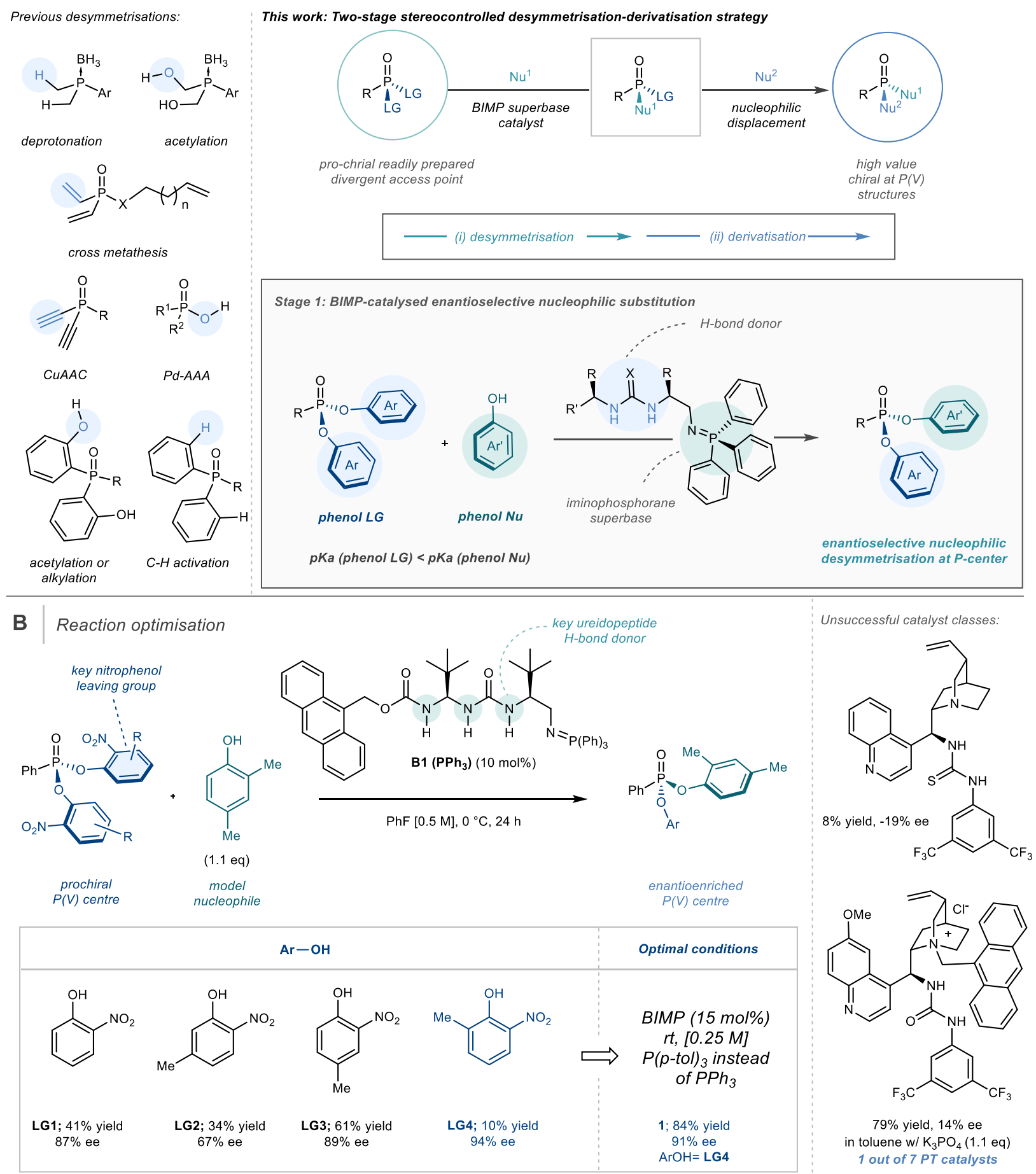

Figure 1. (A) Previous catalysts yielding modestly enantioselective nucleophilic substitution at phosphorous (left) and our reaction design (right). (B) Selected reaction optimisation (left) with representative unsuccessful catalyst classes (right). 
With the optimised conditions in hand, we proceeded to explore the scope of this enantioselective nucleophilic desymmetrisation (Figure 2). We were pleased to find that a variety of phenols possessing ortho-substituents (2-6) were well tolerated, and high yields and enantioselectivities were maintained. Compound 6 (98\% yield, $91 \%$ ee) is of particular interest as a precursor to CycloSal-type prodrugs. ${ }^{40}$ 2,3-Disubstituted phenols were also found to be effective nucleophiles (7). The introduction of electron-withdrawing groups (8) was also possible, despite a slight reduction in enantioselectivity. Bulky, naturally occurring phenols totarol and thymol were also competent nucleophiles for delivering the desymmetrised $\mathrm{P}(\mathrm{V})$ products $(9,10)$ in good yield and high enantioselectivity. Importantly, when totarol was reacted with P1, catalysed by achiral base BEMP, no diastereoselectivity was observed, demonstrating that the stereoselective reaction was under complete catalyst control. Using carvacrol as a nucleophile (11) resulted in diminished enantioselectivity. Nucleophiles bearing no ortho-substituent were found to afford the corresponding products in good yields but poor enantioselectivity confirming that the ortho-substituent was a key discriminating feature in the transition structures governing the stereoselectivity of the reaction (see SI for details). The reaction was also successful on gram-scale $(3 \mathrm{mmol})$ with no loss in yield or enantioselectivity. Desymmetrised product 1 was recrystallized to $>99.9 \%$ ee and the absolute stereochemical configuration was assigned as $(R)$ via single crystal X-ray diffraction analysis (see SI for details). Having probed the scope of the nucleophilic partner, we proceeded to vary the $P$ linked carbon substituent on the phosphonate ester electrophile. Such changes were broadly tolerated with aryl (12), methyl (13), higher alkyl (14-15) and $\beta$-branched (16) substituents maintaining high reaction efficiency and enantioselectivities. Benzyl substituents (17-19) were also tolerated, despite a slight decrease in selectivity. Finally, we were pleased to find that thiophene (20) and (thio)ether $(\mathbf{2 1}, \mathbf{2 2})$ substituted prochiral phosphorous electrophiles were also competent substrates for our methodology. 


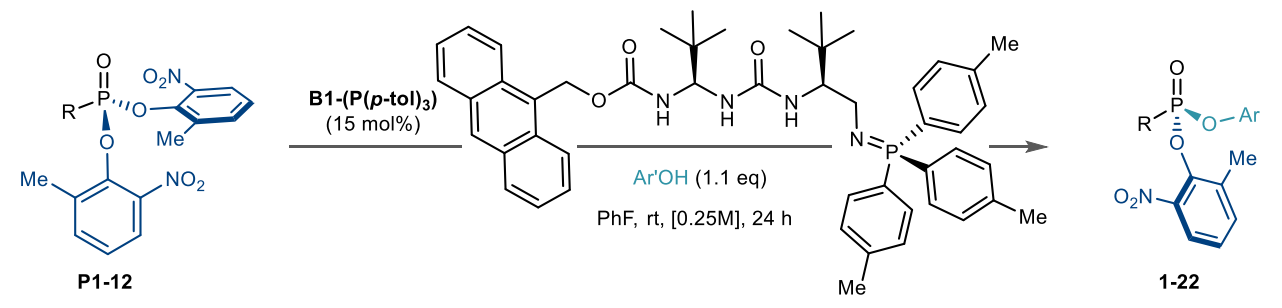

Phenol variation
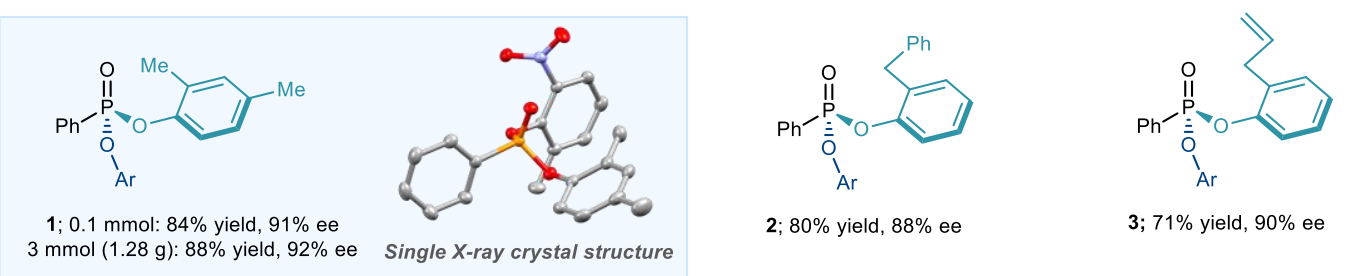

2; $80 \%$ yield, $88 \%$ ee $\quad 3 ; 71 \%$ yield, $90 \%$ ee

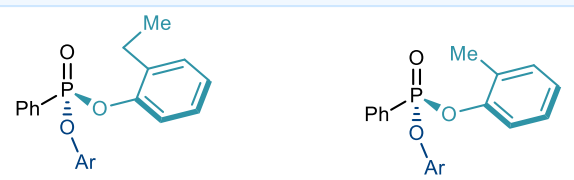

4; $70 \%$ yield, $91 \%$ ee

$5 ; 82 \%$ yield, $82 \%$ ee

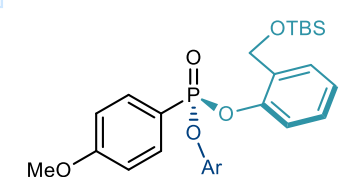

6; $98 \%$ yield, $91 \%$ ee

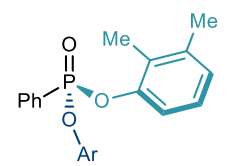

7; $93 \%$ yield, $88 \%$ ee<smiles>COc1ccc(OP(N)(=O)O)c(Cl)c1</smiles>

8; $61 \%$ yield, $75 \%$ ee

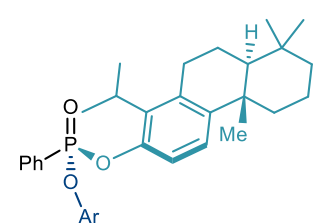

9; $63 \%$ yield, $97: 3 \mathrm{dr}$

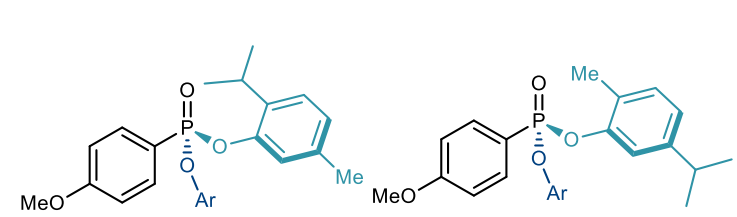

$10 ; 64 \%$ yield, $96 \%$ ee from thymol

11; $50 \%$ yield, $70 \%$ ee from carvacrol

Backbone variation

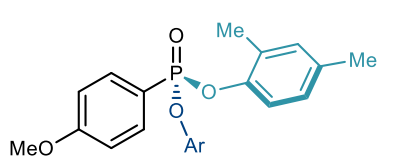

12; $69 \%$ yield, $97 \%$ ee<smiles>Cc1ccc(OP(=O)(Cc2ccccc2)O[Al])c(C)c1</smiles>

$17 ; 73 \%$ yield, $78 \%$ ee

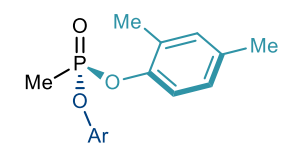

13; $71 \%$ yield, $84 \%$ ee

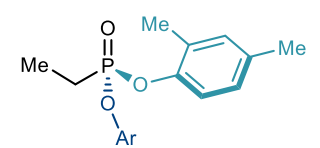

$14 ; 63 \%$ yield, $93 \%$ ee

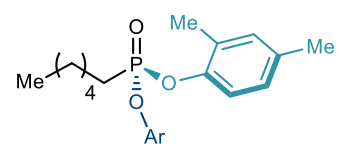

15; $60 \%$ yield, $94 \%$ ee

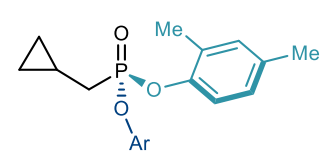

16; $46 \%$ yield, $88 \%$ ee

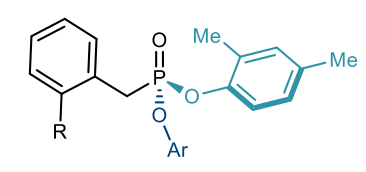

18; R=Me: $42 \%$ yield, $86 \%$ ee 19; $R=\mathrm{CF}_{3}: 43 \%$ yield, $78 \%$ ee

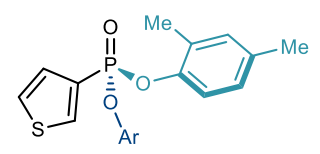

20; $75 \%$ yield, $92 \%$ ee

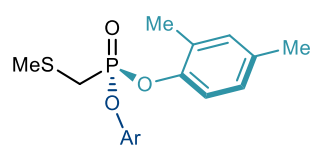

21; $77 \%$ yield, $92 \%$ ee

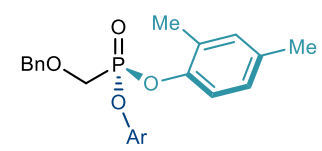

22; $72 \%$ yield, $83 \%$ ee

Figure 2. Scope of the desymmetrisation reaction for the nucleophilic component (top) and electrophilic component (bottom).

Having established the scope of the desymmetrisation for both the nucleophilic and electrophilic components, we proceeded to assess the crucial second stage of the strategy: the enantiospecific nucleophilic substitution of the remaining nitrophenol leaving group (Figure 3). After a preliminary investigation into the applicability of existing protocols, we found that 
Lewis acid activation of the $\mathrm{P}(\mathrm{V})$ species, pioneered by Merck, ${ }^{41}$ was highly efficient in promoting the second nucleophilic displacement. Indeed, treating compound $\mathbf{1}$, with 1.5 equivalent each of $t-\mathrm{BuMgCl}$ and benzyl alcohol in THF at room temperature smoothly afforded mixed phosphonate ester $\mathbf{2 3}$ in almost quantitative yield and 100\% enantiospecificity. Encouraged by this success, we proceeded to use more complex and biologically relevant alcohols as nucleophiles. All four DNA nucleosides (24-27) as well as acetal protected uridine (28) and adenosine (29) were successfully phosphorylated at the 5'-OH with moderate to good yields and >95:5 dr. Acetal protected D-glucose (30) and deoxythymidine (31) were both phosphorylated at the more hindered secondary 3'-OH in excellent yields and diastereoselectivity. Having established reactivity with alcohol nucleophiles we proceeded to investigate the stereocontrolled formation of alternative $P$-heteroatom linkages. When using $n$ propane thiol as the nucleophile, however, no desired product was obtained when using $t$ $\mathrm{BuMgCl}$ to promote the reaction, with the main by-product observed being (2-methyl-6nitrophenyl)(propyl)thioether obtained via $\mathrm{S}_{\mathrm{N}} \mathrm{Ar}$. This obstacle was overcome by switching the promoter 3 equivalents of DBU which gave the desired thiophosphonate ester 32 in $59 \%$ yield and $98 \%$ es. We then turned our attention to $N$-centred nucleophiles and were thrilled to find that when using $N$-Boc benzylamine as the nucleophile we could obtain phosphonamidite ester 33 in $83 \%$ yield and $100 \%$ es. Finally, TBS protected phosphonate ester $\mathbf{6}$ was deprotected with TFA and cyclised with $t$-BuMgCl to give CycloSal derivative 34 in $67 \%$ yield and $92 \%$ es over 2 steps. Upon recrystallisation to enantiopurity and single crystal X-ray diffraction analysis (see SI for details), the absolute configuration of $\mathbf{3 4}$ was determined to be $(R)$ showing inversion of configuration ${ }^{18,42-44}$ with respect to the starting material. 

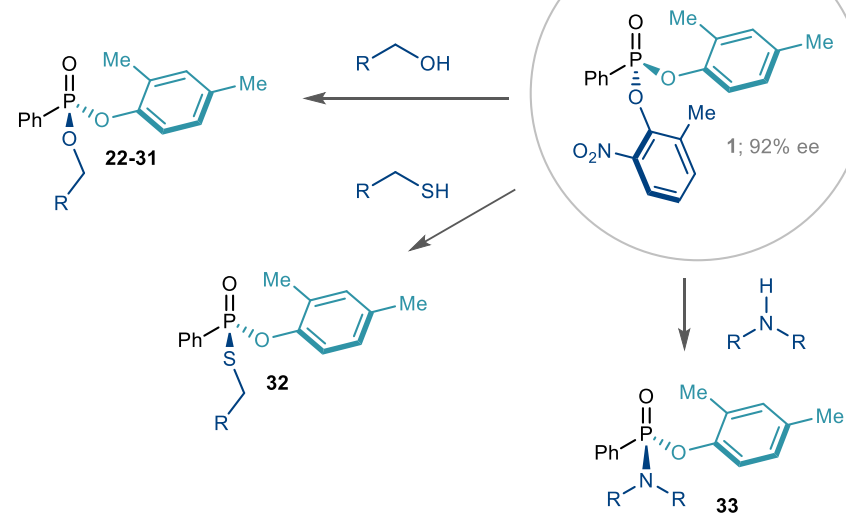

Conditions:

$\mathrm{R}-\mathrm{OH}$ (1.5 eq), $t$-BuMgCl (1.5 eq), THF, rt, 2- $48 \mathrm{~h}$

P-S Bond Formation:

R-SH (3 eq), DBU (3 eq), THF, rt, 2 h

P-N Bond Formation:

Amine (1.5 eq), $t$-BuMgCl (1.5 eq), THF, rt, $2 \mathrm{~h}$

P-O Bond Formation

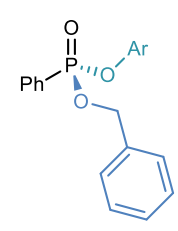

$23,97 \%$ yield, $92 \%$ ee, $100 \%$ es (2 hrs)

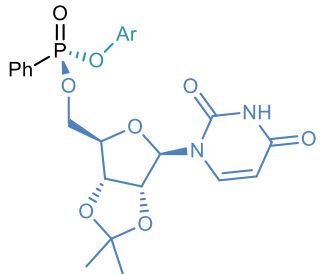

28, $71 \%$ yield, $>95: 5 \mathrm{dr}$ $(2 \mathrm{hrs})^{[\mathrm{a}]}$

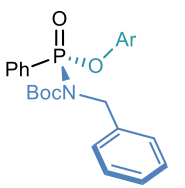

33; $84 \%$ yield, $93 \%$ ee, $100 \%$ es P-N Bond Formation

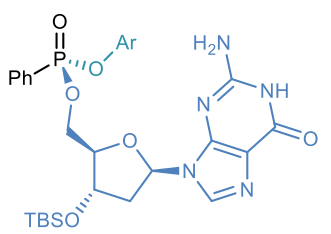

24; $72 \%$ yield, $>95: 5 \mathrm{dr}$ $(16 \mathrm{hrs})^{[\mathrm{a}]}$

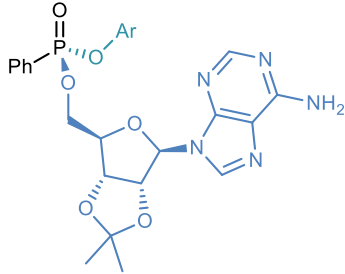

29, $48 \%$ yield, $>95: 5 \mathrm{dr}$ $(16 \mathrm{hrs})^{[\mathrm{a}]}$

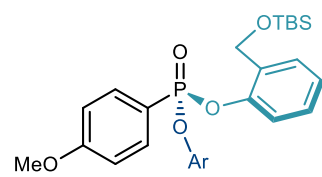

6; $91 \%$ ee

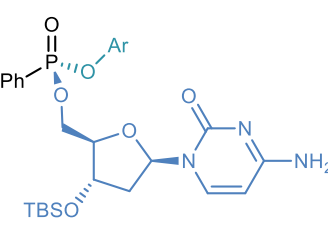

25; $48 \%$ yield, $>95: 5 \mathrm{dr}$ $(48 \mathrm{hrs})^{[\mathrm{b}]}$

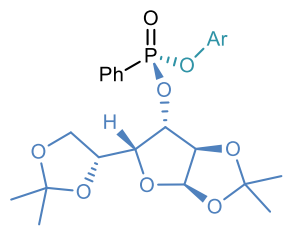

30; $93 \%$ yield, $>95: 5 \mathrm{dr}$ (24 hrs)
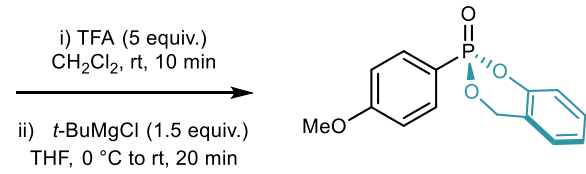

34; $67 \%$ over 2 steps, $84 \%$ ee, $92 \%$ es

CycloSal Derivative

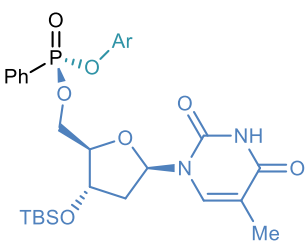

27; $70 \%$ yield, $>95: 5 \mathrm{dr}$ $(48 \mathrm{hrs})^{[\mathrm{a}]}$

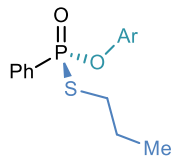

31; $71 \%$ yield, $>95: 5 \mathrm{dr}$ $(16 \mathrm{hrs})^{[\mathrm{a}]}$

32; $59 \%$ yield, $90 \%$ ee, $98 \%$ es P-S Bond Formation

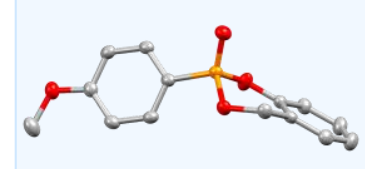

Single $X$-ray crystal structure

Figure 3. Derivatisation of the desymmetrised enantioenriched $\mathrm{P}(\mathrm{V})$ products. $\mathrm{P}-\mathrm{O}$ bond formation (top), $\mathrm{P}-\mathrm{S}$ and $\mathrm{P}-\mathrm{N}$ bond formation (bottom) and synthesis of a CycloSal prodrug derivative (bottom). [a] 3 eq of $t$ - $\mathrm{BuMgCl}$ were used. ${ }^{[b]} 4$ eq of $t-\mathrm{BuMgCl}$ were used.

To confirm the importance of controlling stereochemical configuration at all substitution steps, acetal protected uridine and D-glucose were also reacted with racemic $\mathbf{1}$. Both compounds $\mathbf{2 8}$ and $\mathbf{3 0}$ were obtained as a 1:1 mixture of diastereoisomers, thus highlighting the importance of 
deploying enantioenriched $\mathbf{1}$ in the second nucleophilic substitution to obtain a single diastereomeric product (see SI for details).

To further probe the mechanism and establish the origins of enantioselectivity in the initial nucleophilic desymmetrisation step, a computational Density Functional Theory (DFT) study was undertaken.

In agreement with previous literature reports, calculations on a model system established that the nucleophilic attack and leaving group displacement steps occurred as two discrete steps, with the pentacoordinate intermediate residing in a local minimum (see SI for details). ${ }^{45}$ Building upon this result, a more complex catalytic model was generated. In this model, the chiral backbone, the basic $N$-atom of the iminophosphorane, and the distal benzylic group on of the catalyst were conserved and represented with analogous functional groups as they were found to be essential for achieving high reactivity and enantioselectivity in the key catalystcontrolled desymmetrisation step (Figure 5).

Using this model, it was found that the phosphonate ester can adapt two major orientations upon coordination to the BIMP catalyst, leading to interconvertible and diastereomericallydistinguishable reactant complexes (Figure 5A). By orienting the aryl substituent on the phosphonate ester either pointing away $(\mathbf{S M}-(\boldsymbol{R}))$ or towards $(\mathbf{S M}-(\boldsymbol{S}))$ the BIMP structure allowed for differentiation between the two potential nitro phenol leaving groups in the displacement step and the ensuing formation of the two possible enantiomers. 
The subsequent nucleophilic attack by the deprotonated phenolic nucleophile is the enantiodetermining step, with the transition state for the $(R)$-enantiomer (TS- $(\boldsymbol{R})) 1.5 \mathrm{kcal} \mathrm{mol}^{-}$ ${ }^{1}$ lower in energy than the transition state for the $(S)$-enantiomer (TS- $(S)$ ) (Figure 5, A). Furthermore, the vibrational mode associated with the TS- $(\boldsymbol{R})$ 's imaginary frequency indicates that both the nucleophilic attack and deprotonation events occur concurrently and with a higher degree of synchronicity for the preferred transition state. This energetic difference resulted in a predicted enantiomeric excess of $89 \%$ for the formation of the $(R)$-enantiomer, in good agreement with experimental results. After this step, the reaction is thermodynamically downhill, with the elimination process occurring as a discrete step with a small activation barrier $\left(\Delta \Delta \mathrm{G}^{*}=0.5 \mathrm{kcal} \mathrm{mol}^{-1}\right.$ for the $(R)$-enantiomer and $\Delta \Delta \mathrm{G}^{*}=7.7 \mathrm{kcal} \mathrm{mol}^{-1}$ for the $(S)$ enantiomer). The overall reaction pathway was found to be exergonic and following decomplexation of the desymmetrised product the BIMP catalyst was released back into the cycle.

Further computational analysis of the major enantiomer's transition structure revealed several key substrate-catalyst interactions responsible for stabilising the complex and facilitating the nucleophilic attack (Figure 5, B). For quantitative analysis, bulkier tert-butyl substituents were included in the chiral backbone of the BIMP catalyst. 
A. Reaction energy profile
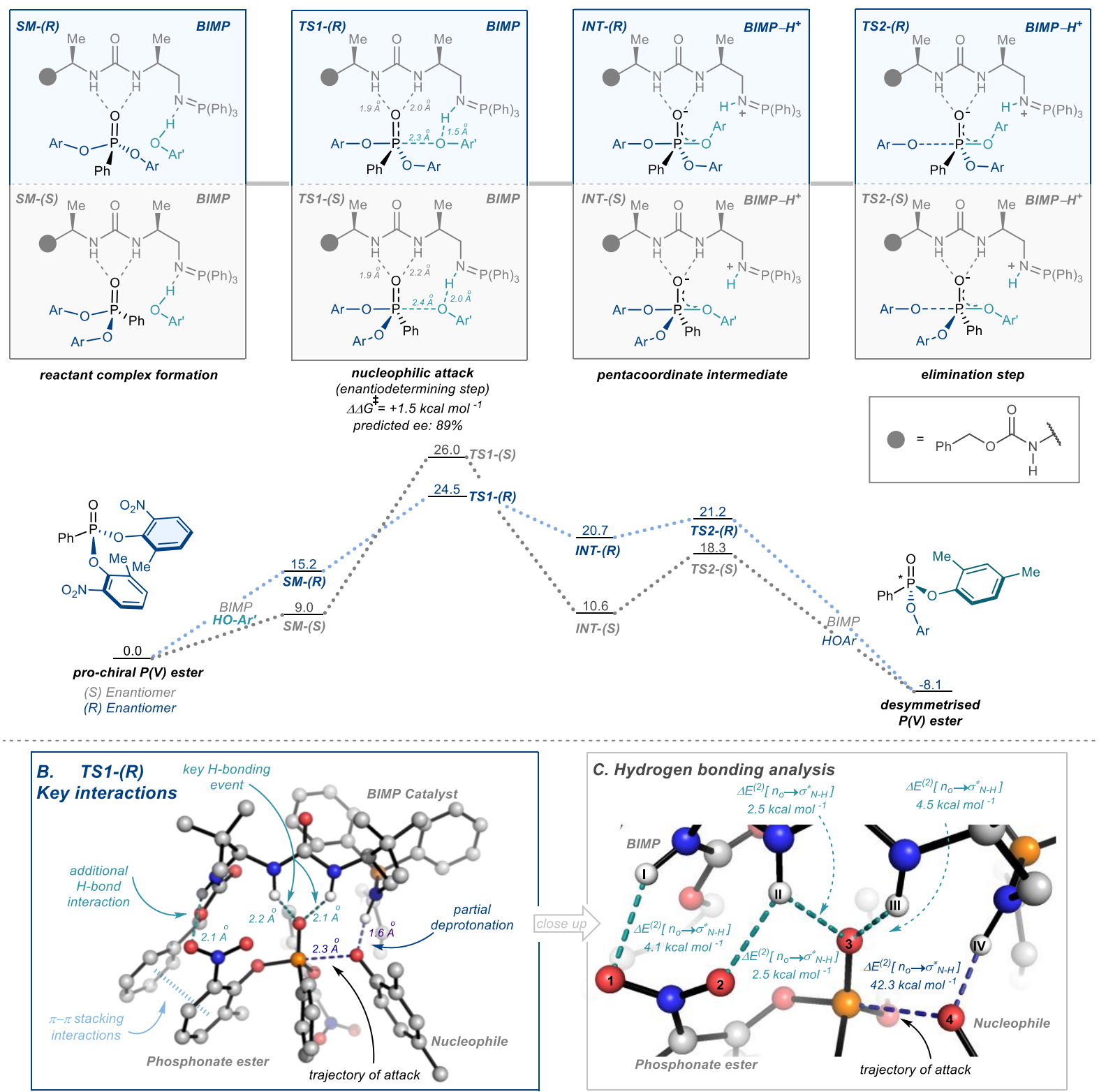

Figure 5. (A) Reaction profile for the enantioselective nucleophilic desymmetrisation of $P(V)$ compounds. A simplified representation of the BIMP organocatalyst was used. The Gibbs free and electronic energies are given in $\mathrm{kcal} \mathrm{mol}^{-1}$ calculated relative to the nucleophile, BIMP catalyst, and pro-chiral phosphonate structures. (B) Proposed transition state structure with full catalytic structure included for the formation of the major stereoisomer (TS1-(R)). (C) Second order perturbation analysis (kcal $\mathrm{mol}^{-1}$ ) of TS1-(R). All geometries were optimised at the SMD(fluorobenzene)-M062X/def2TZVP//SMD(fluorobenzene)-M06-2X/def2SVP level of theory. 
In this model, various hydrogen bonding interactions between the catalyst and substrate were quantified using second order perturbation analysis. In particular, the urea (H(II) and $\mathbf{H}(\mathbf{I I I}))$ and carbamate $(\mathbf{H}(\mathbf{I}))$ portions of the BIMP were found to bind with the $\mathrm{P}=\mathrm{O}(\mathbf{O 3})$ oxygen and nitro (O1 and O2) substituent on the substrate, respectively (Figure 5, C). These interactions not only facilitated binding of the substrate within the chiral scaffold of the catalyst, but also contributed to transition state stabilisation (Figure 5, C). The analysis also revealed that the phenol nucleophile (O4- H(IV)) was deprotonated by the basic iminophosphorane, but the resulting phenolate remained closely associated to the protonated $\mathrm{N}$-atom of the catalyst. Thereby, a more activated species was generated to attack at the phosphorous centre. Moreover, additional $\pi-\pi$ stacking interactions between the outgoing leaving group and the remote benzylic carbamate substituent of the BIMP catalyst stabilised the formation of the pentacoordinate intermediate and positioned the ester substituent for subsequent displacement further along the reaction pathway.

In conclusion, a conceptually novel two-stage strategy for the synthesis of stereogenic $\mathrm{P}(\mathrm{V})$ compounds through an unprecedented enantioselective nucleophilic desymmetrisation and subsequent enantiospecific derivatization, was developed. A novel BIMP catalyst bearing a ureidopeptide H-bond donor moiety provided a unique chiral environment and sufficient pronucleophile / substrate activation to allow the desymmetrisation to proceed with excellent yield and enantioselectivity. Through judicious choice of leaving group, facile downstream diversification of the desymmetrised $\mathrm{P}(\mathrm{V})$ ester with a multitude of medicinally relevant nucleophiles with very high enantiospecificity was allowed. This study represents a clear strategic departure from previously established catalytic methods which rely on substrate engineering and do not easily allow for facile downstream modification to medicinally attractive molecules. Finally, DFT studies were carried out to elucidate an energetically plausible reaction pathway for the two enantiomeric products and to uncover the main attractive 
non-covalent interactions responsible for the high levels of enantioselectivity observed for the major enantiomer. This conceptually novel, two-stage desymmetrisation/derivatisation approach has enabled modular catalytic enantioselective access to stereogenic-P(V) compounds via a strategy which we hope will unlock new opportunities and inspire further developments in the field.

Data Availability: Crystallographic data are available free of charge from the Cambridge Crystallographic Data Centre under reference CCDC 2097043 (1) and CCDC 2097044 (34). Additional optimisation data, full synthetic methods, and characterisation data are available in the supplementary materials.

\section{References:}

1. Rodriguez, J. B. \& Gallo-Rodriguez, C. The Role of the Phosphorus Atom in Drug Design. ChemMedChem 14, 190-216 (2018).

2. Siegel, D. et al. Therapeutic efficacy of the small molecule GS-5734 against Ebola virus in rhesus monkeys. Nature 531, 381-385 (2016).

3. Dutartre, M., Bayardon, J. \& Jugé, S. Applications and stereoselective syntheses of P-chirogenic phosphorus compounds. Chem. Soc. Rev. 45, 5771-5794 (2016).

4. Liu, S. et al. First catalytic enantioselective synthesis of P-stereogenic phosphoramides via kinetic resolution promoted by a chiral bicyclic imidazole nucleophilic catalyst. Tetrahedron Asymmetry $\mathbf{2 3}, 329$ 332 (2012).

5. Tamura, T.; Ryukoku, E. Asymmetric synthesis of organic phosphorus compounds. JP 2003128688 A, (2003)

6. Wang, L. et al. Organocatalytic Enantioselective Synthesis of P-Stereogenic Chiral Oxazaphospholidines. European J. Org. Chem. 2016, 2024-2028 (2016).

7. Borissov, A. et al. Organocatalytic enantioselective desymmetrisation. Chem. Soc. Rev. 45, 5474-5540 (2016)

8. Núñez, M. G., Farley, A. J. M. \& Dixon, D. J. Bifunctional iminophosphorane organocatalysts for 
enantioselective synthesis: Application to the ketimine nitro-Mannich reaction. J. Am. Chem. Soc. 135, 16348-16351 (2013).

9. Farley, A. J. M., Sandford, C. \& Dixon, D. J. Bifunctional Iminophosphorane Catalyzed Enantioselective Sulfa-Michael Addition to Unactivated $\alpha$-Substituted Acrylate Esters. J. Am. Chem. Soc. 137, 1599215995 (2015).

10. Formica, M., Rozsar, D., Su, G., Farley, A. J. M. M. \& Dixon, D. J. Bifunctional Iminophosphorane Superbase Catalysis: Applications in Organic Synthesis. Acc. Chem. Res. 53, 2235-2247 (2020).

11. Mehellou, Y., Rattan, H. S. \& Balzarini, J. The ProTide Prodrug Technology: From the Concept to the Clinic. J. Med. Chem. 61, 2211-2226 (2018).

12. DiRocco, D. A. et al. A multifunctional catalyst that stereoselectively assembles prodrugs. Science 356, 426-430 (2017).

13. Knouse, K. W. et al. Unlocking P(V): Reagents for chiral phosphorothioate synthesis. Science 361, 12341238 (2018).

14. Juge, S., Stephan, M., Laffitte, J. A. \& Genet, J. P. Efficient asymmetric synthesis of optically pure tertiary mono and diphosphine ligands. Tetrahedron Lett. 31, 6357-6360 (1990).

15. Juge, S. \& Genet, J. P. Asymmetric synthesis of phosphinates, phosphine oxides and phosphines by Michaelis Arbuzov rearrangement of chiral oxazaphospholidine. Tetrahedron Lett. 30, 2783-2786 (1989).

16. Corey, E. J., Chen, Z. \& Tanoury, G. J. A New and Highly Enantioselective Synthetic Route to P-Chiral Phosphines and Diphosphines. J. Am. Chem. Soc. 115, 11000-11001 (1993).

17. Han, Z. S. et al. Efficient asymmetric synthesis of $\mathrm{P}$-chiral phosphine oxides via properly designed and activated benzoxazaphosphinine-2-oxide agents. J. Am. Chem. Soc. 135, 2474-2477 (2013).

18. Kuwabara, K., Maekawa, Y., Minoura, M., Maruyama, T. \& Murai, T. Chemoselective and Stereoselective Alcoholysis of Binaphthyl Phosphonothioates: Straightforward Access to Both Stereoisomers of Biologically Relevant P -Stereogenic Phosphonothioates. J. Org. Chem. 85, 1444614455 (2020).

19. Xu, D. et al. Enantiodivergent Formation of C-P Bonds: Synthesis of P-Chiral Phosphines and Methylphosphonate Oligonucleotides. J. Am. Chem. Soc. 142, 5785-5792 (2020). 
20. Beaud, R., Phipps, R. J. \& Gaunt, M. J. Enantioselective Cu-Catalyzed Arylation of Secondary Phosphine Oxides with Diaryliodonium Salts toward the Synthesis of P-Chiral Phosphines. J. Am. Chem. Soc. 138, 13183-13186 (2016).

21. Dai, Q., Li, W., Li, Z. \& Zhang, J. P-Chiral Phosphines Enabled by Palladium/Xiao-Phos-Catalyzed Asymmetric P-C Cross-Coupling of Secondary Phosphine Oxides and Aryl Bromides. J. Am. Chem. Soc. 141, 20556-20564 (2019).

22. Liu, X., Zhang, Y.-Q., Han, X., Sun, S. \& Zhang, Q. Ni-Catalyzed Asymmetric Allylation of Secondary Phosphine Oxides. J. Am. Chem. Soc. 141, 16584-16589 (2019).

23. Diesel, J. \& Cramer, N. Generation of Heteroatom Stereocenters by Enantioselective C-H Functionalization. ACS Catal. 9, 9164-9177 (2019).

24. Genov, G. R., Douthwaite, J. L., Lahdenperä, A. S. K., Gibson, D. C. \& Phipps, R. J. Enantioselective remote $\mathrm{C}-\mathrm{H}$ activation directed by a chiral cation. Science $\mathbf{3 6 7}, 1246-1251$ (2020).

25. Huang, Q.-H. et al. Access to P-Stereogenic Compounds via Desymmetrizing Enantioselective Bromination. Chem. Sci. 68, 42-61 (2021).

26. Wiktelius, D., Johansson, M. J., Luthman, K. \& Kann, N. A Biocatalytic Route to P-Chirogenic Compounds by Lipase-Catalyzed Desymmetrization of a Prochiral Phosphine-Borane. Org. Lett. 7, 4991-4994 (2005).

27. Toda, Y., Pink, M. \& Johnston, J. N. Brønsted acid catalyzed phosphoramidic acid additions to alkenes: Diastereo- and enantioselective halogenative cyclizations for the synthesis of c - And P -chiral phosphoramidates. J. Am. Chem. Soc. 136, 14734-14737 (2014).

28. Harvey, J. S. et al. Enantioselective synthesis of P-stereogenic phosphinates and phosphine oxides by molybdenum-catalyzed asymmetric ring-closing metathesis. Angew. Chem. Int. Ed. 48, 762-766 (2009).

29. Trost, B. M., Spohr, S. M., Rolka, A. B. \& Kalnmals, C. A. Desymmetrization of Phosphinic Acids via Pd-Catalyzed Asymmetric Allylic Alkylation: Rapid Access to P-Chiral Phosphinates. J. Am. Chem. Soc. 141, 14098-14103 (2019).

30. Zhu, R.-Y., Chen, L., Hu, X.-S., Zhou, F. \& Zhou, J. Enantioselective synthesis of P -chiral tertiary phosphine oxides with an ethynyl group via $\mathrm{Cu}$ ( i )-catalyzed azide-alkyne cycloaddition. Chem. Sci. 11, 97-106 (2020). 
31. Yang, G., Li, Y., Li, X. \& Cheng, J.-P. Access to P-chiral phosphine oxides by enantioselective allylic alkylation of bisphenols. Chem. Sci. 10, 4322-4327 (2019).

32. Zheng, Y., Guo, L. \& Zi, W. Enantioselective and Regioselective Hydroetherification of Alkynes by GoldCatalyzed Desymmetrization of Prochiral Phenols with P-Stereogenic Centers. Org. Lett. 20, 7039-7043 (2018).

33. Zhang, Y. et al. Asymmetric Synthesis of P-Stereogenic Compounds via Thulium(III)-Catalyzed Desymmetrization of Dialkynylphosphine Oxides. ACS Catal. 9, 4834-4840 (2019).

34. Lemouzy, S., Giordano, L., Hérault, D. \& Buono, G. Introducing Chirality at Phosphorus Atoms: An Update on the Recent Synthetic Strategies for the Preparation of Optically Pure P-Stereogenic Molecules. Eur. J. Org. Chem. 2020, 3351-3366 (2020).

35. Wang, Y.-H. et al. Activating Pronucleophiles with High p K a Values: Chiral Organo-Superbases. Angew. Chem. Int. Ed. 59, 8004-8014 (2020).

36. Greenhalgh, M. D., Qu, S., Slawin, A. M. Z. \& Smith, A. D. Multiple roles of aryloxide leaving groups in enantioselective annulations employing $\alpha, \beta$-unsaturated acyl ammonium catalysis. Chem. Sci. 9, 49094918 (2018).

37. Diosdado, S. et al. Catalytic Enantioselective Synthesis of Tertiary Thiols From 5 H -Thiazol-4-ones and Nitroolefins: Bifunctional Ureidopeptide-Based Brønsted Base Catalysis. Angew. Chem. Int. Ed. 52, 11846-11851 (2013).

38. Diosdado, S., Lõpez, R. \& Palomo, C. Ureidopeptide-based Brønsted bases: Design, synthesis and application to the catalytic enantioselective synthesis of $\beta$-amino nitriles from (arylsulfonyl)acetonitriles. Chem. Eur. J. 20, 6526-6531 (2014).

39. Vera, S. et al. Synthesis of $\beta$-Hydroxy $\alpha$-Amino Acids Through Brønsted Base-Catalyzed syn -Selective Direct Aldol Reaction of Schiff Bases of Glycine o -Nitroanilide. J. Org. Chem. 86, 7757-7772 (2021).

40. Meier, C. cycloSal phosphates as chemical trojan horses for intracellular nucleotide and glycosylmonophosphate delivery - Chemistry meets biology. Eur. J. Org. Chem. 1081-1102 (2006).

41. Simmons, B., Liu, Z., Klapars, A., Bellomo, A. \& Silverman, S. M. Mechanism-Based Solution to the ProTide Synthesis Problem: Selective Access to Sofosbuvir, Acelarin, and INX-08189. Org. Lett. 19, 2218-2221 (2017). 
42. Asymmetric Synthesis of P-Chirogenic Phosphorus Compounds. in Asymmetric Synthesis in Organophosphorus Chemistry 35-99 (Wiley-VCH Verlag GmbH \& Co. KGaA, 2016).

43. Van Bochove, M. A., Swart, M. \& Bickelhaupt, F. M. Nucleophilic substitution at phosphorus (SN2@P): Disappearance and reappearance of reaction barriers. J. Am. Chem. Soc. 128, 10738-10744 (2006).

44. Kolodiazhnyi, O. I. \& Kolodiazhna, A. Nucleophilic substitution at phosphorus: stereochemistry and mechanisms. Tetrahedron: Asymmetry 28, 1651-1674 (2017).

45. Kirby, A. J. \& Nome, F. Fundamentals of Phosphate Transfer. Acc. Chem. Res. 48, 1806-1814 (2015).

\section{Acknowledgements}

The authors thank Ms. Shree Sowndarya for preliminary computational experiments. M. F. and T. R. are grateful to the EPSRC Centre for Doctoral Training in Synthesis for Biology and Medicine (EP/L015838/1) for studentships, generously supported by AstraZeneca, Diamond Light Source, Defence Science and Technology Laboratory, Evotec, GlaxoSmithKline, Janssen, Novartis, Pfizer, Syngenta, Takeda, UCB and Vertex. T. R. also thanks the Royal Commission of 1851 Industrial Fellowship and NSERC PGS-D. N. S is grateful to the JSPS Overseas Challenge Program for Young Researchers.

\section{Author contributions}

M. F. and D. J. D. conceived the project. M. F., H. S. and N. S. conducted all experimental work and analysed the data. A. J. M. F. designed and first synthesised the ureidopeptide BIMP catalyst. T. R. conducted all computational work under the guidance of F. D.. K. E. C. conducted single-crystal X-Ray diffraction experiments. M. F. wrote the manuscript with contributions and proof-reading from all authors. D. J. D. directed the project.

\section{Competing interests}

Authors declare no competing interests. 


\section{Additional information}

Correspondence and requests for materials should be addressed to F.D. \& D.J.D. 